Article

\title{
Conformally Coupled Inflation
}

\section{Valerio Faraoni}

Physics Department and STAR Research Cluster, Bishop's University, 2600 College St., Sherbrooke, Québec J1M 1Z7, Canada; E-Mail: vfaraoni@ubishops.ca; Tel.: +1-819-822-9600 (ext. 2490); Fax: +1-819-822-9611

Received: 29 June 2013; in revised form: 19 July 2013 / Accepted: 19 July 2013 /

Published: 30 July 2013

\begin{abstract}
A massive scalar field in a curved spacetime can propagate along the light cone, a causal pathology, which can, in principle, be eliminated only if the scalar couples conformally to the Ricci curvature of spacetime. This property mandates conformal coupling for the field driving inflation in the early universe. During slow-roll inflation, this coupling can cause super-acceleration and, as a signature, a blue spectrum of primordial gravitational waves.
\end{abstract}

Keywords: inflation; non-minimal coupling; early universe

\section{Introduction}

A period of inflationary expansion of the early universe has gradually become to be accepted by most cosmologists as a paradigm of the modern scientific picture of the universe's history. Although there is no direct proof that inflation actually occurred, and it is healthy to contemplate alternatives, such as bouncing models $[1,2]$, the ekpyrotic universe [3-5] or string gas cosmology [6-8], the temperature anisotropies discovered by the COBE satellite and further studied by the WMAP and PLANCK missions have a spectrum close to the Harrison-Zel'dovich one predicted by inflation, which certainly is some support for the view of an inflationary early universe.

Assuming that inflation occurred early on and that it was driven by some scalar field, $\phi$ (arguably the simplest, although not mandatory, class of inflationary scenarios), research has for long focused on identifying specific scenarios of inflation corresponding to particular choices of the scalar field potential, $V(\phi)$, motivated by particle physics. Here, we argue that the scalar field, $\phi$, driving inflation, should be non-minimally (in fact, conformally) coupled to the Ricci curvature of spacetime, $R$, in order to 
avoid causal pathologies. Conformal (or, in general, non-minimal) coupling was originally introduced in radiation problems [9] or in the renormalization of scalar fields in curved backgrounds [10-16]. Therefore, it certainly is not obvious that a conventional minimally coupled scalar (with timelike or null gradient) can suffer from light cone pathologies, but this is indeed the case, as was pointed out long ago for test fields [17]. Let us revisit the argument and its consequences for inflation.

\section{Non-Minimal Coupling}

A scalar field, $\phi$, with mass, $m$, propagating in curved spacetime satisfies the Klein-Gordon equation:

$$
\square \phi-m^{2} \phi-\xi R \phi=0
$$

where the dimensionless non-minimal coupling constant, $\xi$, between the scalar and the Ricci curvature is here allowed for generality (we will see that minimal coupling, corresponding to $\xi=0$, is, in fact, ruled out). Here, $\square=g^{\mu \nu} \nabla_{\mu} \nabla_{\nu}$, where $g_{\mu \nu}$ is the spacetime metric and $\nabla_{\mu}$ is its covariant derivative operator. Consider the solution of Equation (1) corresponding to a delta-like source, which is nothing but the Green function, $G_{R}\left(x^{\prime}, x\right)$, of this equation:

$$
\left[g^{\mu^{\prime} \nu^{\prime}}\left(x^{\prime}\right) \nabla_{\mu^{\prime}} \nabla_{\nu^{\prime}}-m^{2}-\xi R\left(x^{\prime}\right)\right] G_{R}\left(x^{\prime}, x\right)=-\delta\left(x^{\prime}, x\right)
$$

where $\delta\left(x^{\prime}, x\right)$ is the spacetime delta. By imposing the usual boundary conditions, we are restricted to the retarded Green function. It is then well known $[18,19]$ that the retarded Green function, $G_{R}$, can be split as:

$$
G_{R}\left(x^{\prime}, x\right)=\Sigma\left(x^{\prime}, x\right) \delta_{R}\left[\Gamma\left(x^{\prime}, x\right)\right]+W\left(x^{\prime}, x\right) \Theta\left[-\Gamma\left(x^{\prime}, x\right)\right]
$$

where $\Gamma\left(x^{\prime}, x\right)$ is the square of the proper distance between $x$ and $x^{\prime}$ calculated along the geodesic connecting these two spacetime points (which is unique in a normal domain), $\delta_{R}(\Gamma)$ is the usual Dirac delta and $\Theta(-\Gamma)$ is the Heaviside step function with support in the past of $x$. The first term on the right hand side of Equation (3) describes a contribution to $\phi(x)$ coming from the past light cone of $x$, while the second term describes a contribution from the interior of this light cone. The functions, $\Sigma$ and $W$, are coefficients.

If the curved spacetime manifold is to be approximated by its tangent space (which, loosely speaking, is the spirit of the Equivalence Principle of relativity), in the limit, $x^{\prime} \rightarrow x$, in which the two points coincide, the Green function must reduce to the one of Minkowski space [17], i.e., it must be:

$$
\Sigma\left(x^{\prime}, x\right) \rightarrow \Sigma_{M}\left(x^{\prime}, x\right)=\frac{1}{4 \pi}, \quad W\left(x^{\prime}, x\right) \rightarrow W_{M}\left(x^{\prime}, x\right)
$$

as $x^{\prime} \rightarrow x$. It is rather straightforward to expand all these functions in this limit, obtaining [17,18,20,21]:

$$
\begin{aligned}
\Sigma\left(x^{\prime}, x\right) & =\frac{1}{4 \pi}+\mathrm{O}\left(x^{\prime}, x\right) \\
W\left(x^{\prime}, x\right) & =-\frac{1}{8 \pi}\left[m^{2}+\left(\xi-\frac{1}{6}\right) R(x)\right]+\mathrm{O}\left(x^{\prime}, x\right) \\
W_{M}\left(x^{\prime}, x\right) & =-\frac{m^{2}}{8 \pi}+\mathrm{O}\left(x^{\prime}, x\right)
\end{aligned}
$$


where $\mathrm{O}\left(x^{\prime}, x\right)$ generically denotes terms, which vanish as $x^{\prime} \rightarrow x$. Backscattering of the scalar, $\phi$, can be due to both a non-vanishing mass, $m$, or to the background curvature appearing in the term, $-\left(\xi-\frac{1}{6}\right) \frac{R(x)}{8 \pi}$ in Equation (6). If $m \neq 0$, at spacetime points where

$$
m^{2}+\left(\xi-\frac{1}{6}\right) R(x)=0
$$

a massive scalar, $\phi$, will propagate strictly along the light cone, which is clearly a causal pathology. It is even possible to concoct a space of constant curvature, $R$, such that the backscattering tail, due to the curvature, $\left[-\left(\xi-\frac{1}{6}\right) \frac{R(x)}{8 \pi}\right]$, exactly compensates the tail, $\left[-\frac{m^{2}}{8 \pi}\right]$, due to the mass, $m$. This pathology is possible for $\xi=0$. Indeed, the only way to eliminate this disturbing possibility is to have $\xi=1 / 6$ (conformal coupling); then, the propagation of a massive $\phi$ is forced to be inside the light cone.

Note that conformal invariance has not been imposed or implied in any way. It is obtained simply to avoid causal pathologies. The physical interpretation of the result is the following: because only propagation along the light cone is involved in the argument, there must be no scale in the physics of the scalar field, which implies conformal invariance.

If the argument above applies to a free test field, it will also apply to a scalar field in a generic potential, $V(\phi)$, and to a gravitating scalar field, which always has the previous case as a limit.

Let us review briefly the various formulations of the Equivalence Principle. The Weak Equivalence Principle (WEP) states that if an uncharged test body is at an initial spacetime point with an initial four-velocity, its subsequent trajectory will not depend on its internal structure and composition.

The Einstein Equivalence Principle (EEP) states that (a) WEP holds, (b) the outcome of any local non-gravitational test experiment is independent of the velocity of the freely falling apparatus (Local Lorentz Invariance, LLI) and (c) the outcome of any local non-gravitational test experiment is independent of where and when in the universe it is performed (Local Position Invariance, LPI).

The Strong Equivalence Principle (SEP) consists of: (a) WEP holds for self-gravitating bodies, as well as for test bodies; (b) the outcome of any local test experiment is independent of the four-velocity of the freely falling apparatus (Local Lorentz Invariance, LLI); and (c) the outcome of any local test experiment is independent of where and when in the universe it is performed (Local Position Invariance, LPI).

The WEP is a statement about mechanics: it requires only the existence of preferred trajectories, the free fall trajectories followed by test particles, and these curves are the same independently of the mass and internal composition of the particles that follow them (universality of free fall). By itself, WEP does not imply the existence of a metric or of geodesic curves (this requirement arises only through the EEP by combining the WEP with requirements (b) and (c) [22]. The EEP extends the WEP to all areas of non-gravitational physics. The SEP further extends the WEP to self-gravitating bodies and requires LLI and LPI to hold also for gravitational experiments, in contrast to the EEP. All versions of the Equivalence Principle have been subjected to experimental verification, but, thus far, stringent tests only exist for the WEP and the EEP [22].

Originally [17], the argument for $\xi=1 / 6$ was presented as enforcing the EEP [22] applied to a test or a gravitating field, $\phi$. A posteriori, however, there is no need to invoke the Equivalence Principle, and $\phi$ could be a gravitational scalar field (for example, in a scalar-tensor theory of gravity), about which the EEP has nothing to say. Although the argument supporting the value, 1/6, of the coupling constant, 
$\xi$ (rather than the value, $\xi=0$ ), relies only on the absence of causal pathologies in the propagation of $\phi$-waves, it is interesting to elaborate on it in light of the recent paper [23] on theories of gravity satisfying the SEP. The author of [23] looks for ways to implement the SEP on theories of gravity and, on the basis of the analogy with the Standard Model of particle physics, concludes that the SEP is embodied by the condition on the Riemann tensor:

$$
\nabla_{\sigma} R_{\lambda \mu \nu}^{\sigma}=0
$$

which is analogous to the condition:

$$
D_{\mu} F^{\mu \nu}=0
$$

for non-Abelian Yang-Mills fields of strength, $F^{\mu \nu}$, which satisfy $\left[D_{\mu}, D_{\nu}\right]=i F_{\mu \nu}$ (where $D_{\mu}$ is the covariant derivative). The Riemann tensor satisfies the analogous relation:

$$
\left[\nabla_{\mu}, \nabla_{\nu}\right]_{\beta}^{\alpha}=-R_{\beta \mu \nu}^{\alpha}
$$

(This characterization of the SEP, however, is different from the traditional one of, e.g., [22], presented above.) Equation (9) expresses the condition that "gravitons gravitate the same way that gluons glue" [23]. What is interesting for us is that, by considering general scalar-tensor theories of gravity described by the (Jordan frame) action:

$$
S_{S T}=\frac{1}{16 \pi} \int d^{4} x \sqrt{-g}\left[\phi R-\frac{\omega(\phi)}{\phi} g^{\mu \nu} \nabla_{\mu} \phi \nabla_{\nu} \phi\right]+S^{(\text {matter })}
$$

where the Brans-Dicke-like $\phi$ is of gravitational nature (we use units in which Newton's constant, $G$, and the speed of light, $c$, are unity and the Brans-Dicke coupling, $\omega(\phi)$, is a function of $\phi$ ). In general, the gravitational or non-gravitational nature of a field depends on the conformal frame representation of the theory; see the discussion in [24]. In short, scalar-tensor gravity can be discussed in the Jordan frame (meaning the set of variables, $\left(g_{\mu \nu}, \phi\right)$ ), in which the scalar field, $\phi$, couples explicitly to the Ricci curvature and matter is minimally coupled (which has the consequence that massive test particles follow timelike geodesics). Alternatively, one can describe the theory in the Einstein conformal frame, the set of variables, $\left(\tilde{g}_{\mu \nu}, \tilde{\phi}\right)$, related to the Jordan frame by the conformal redefinition of the metric:

$$
g_{\mu \nu} \longrightarrow \tilde{g}_{\mu \nu}=\phi g_{\mu \nu}
$$

and the non-linear field redefinition:

$$
d \tilde{\phi}=\sqrt{\frac{2 \omega(\phi)+3}{16 \pi}} \frac{d \phi}{\phi}
$$

In the Einstein frame, the scalar field has canonical kinetic energy and couples minimally to gravity (i.e., there is no explicit coupling between $\phi$ and $R$ ), but it couples directly to the the matter Lagrangian in the action. As a consequence, uncharged particles in the Einstein frame do not follow geodesics of the metric, $\tilde{g}_{\mu \nu}$, but deviate from them, due to a force proportional to the gradient of the scalar field. Massless particles, the physics of which is conformally invariant, follow null geodesics in both frames (e.g., [25]). 
It turns out that imposing the SEP condition (9) selects only two possible theories [23]. These are Nordstrom's scalar gravity (in which the metric is conformally flat and there is only a scalar degree of freedom) and the theory with:

$$
\omega(\phi)=\frac{3 \phi}{2(\phi-1)}
$$

In the latter case, the field redefinition, $\phi \rightarrow \varphi$, with:

$$
\phi=1-\frac{4 \pi \varphi^{2}}{3}
$$

recasts the action as:

$$
S=\frac{1}{16 \pi} \int d^{4} x \sqrt{-g}\left[\left(\frac{1}{2}-\frac{\varphi^{2}}{12}\right) R-\frac{1}{2} g^{\mu \nu} \nabla_{\mu} \varphi \nabla_{\nu} \varphi\right]+S^{(\text {matter })}
$$

which is the action for a conformally coupled scalar field. In other words, insisting that the gravitational Brans-Dicke-like scalar field, $\phi$, satisfies the EEP (or that the theory satisfies the SEP), leading to the requirement that it be conformally coupled. The traditional SEP amounts to imposing that the Weak Equivalence Principle of mechanics is satisfied also by gravitating bodies, plus local Lorentz invariance and local position invariance [22]. Following the definition of SEP adopted in [23], it would seem that the SEP would correspond to imposing the EEP also on gravitational fields.

Now, if $\phi$ is a gravitational scalar field in a theory of gravity alternative to general relativity, there is no reason for it to satisfy the EEP. Moreover, the Brans-Dicke-like field of scalar-tensor gravity is not supposed to be the one driving inflation - even in the extended and hyperextended inflationary scenarios based on Brans-Dicke gravity and on more general scalar-tensor theories, respectively; it is a second non-gravitational scalar field that is responsible for inflation (see, e.g., the review in [25]). However, any field satisfying Equation (1) should be conformally coupled, $\xi=1 / 6$. Let us review the consequences of conformal coupling if $\phi$ is the scalar field driving inflation in the early universe.

\section{Consequences for Inflation}

It is well known that, if one quantizes a scalar field on a curved background, a non-minimal coupling to the Ricci scalar, $R$, is introduced, even if it was absent in the classical theory [10-16]. In asymptotically free grand unified theories, depending on the gauge group and the matter content, $\xi$ is a running coupling and, generically, $1 / 6$ is a stable infrared fixed point [26-33]. According to the previous (classical) argument, the inflation field fueling inflation should be coupled conformally. Then, one should revisit inflation, keeping in mind that conformal coupling is not an option, but is required for consistency of the theory. Over the years, several authors have studied non-minimally coupled inflatons, usually in a rather opportunistic way, i.e., the coupling constant, $\xi$, was usually considered as a free parameter to be adjusted at will in order to alleviate fine-tuning problems in the potential [34-50]. Now the value of $\xi$ is forced upon us. It has been demonstrated that viable scenarios of inflation for an unperturbed universe can occur with non-minimal coupling [34-50]. A possible obstacle is the fact that the effective term, $-\xi R \phi^{2} / 2$, in the Lagrangian could, in principle, spoil the flatness of an inflationary potential, $V(\phi)$ [34-50], but this difficulty is not crucial. What is more, new features of the dynamics emerge, which are not possible when $\xi=0$ [51,52]. By adopting a spatially flat Friedmann-Lemâitre-Robertson-Walker metric:

$$
d s^{2}=-d t^{2}+a^{2}(t)\left(d x^{2}+d y^{2}+d z^{2}\right)
$$


the field equations are:

$$
\begin{aligned}
& H^{2}=\frac{\kappa}{3} \rho \\
& \frac{\ddot{a}}{a}=\dot{H}+H^{2}=-\frac{\kappa}{6}(\rho+P) \\
& \ddot{\phi}+3 H \dot{\phi}+\frac{d V}{d \phi}+\xi R \phi=0
\end{aligned}
$$

where $\rho$ and $P$ are the energy density and pressure of the cosmic fluid, respectively, $\kappa \equiv 8 \pi G$ ( $G$ being Newton's constant) and an overdot denotes differentiation with respect to the comoving time, $t$. Equations (19) and (20) yield:

$$
\dot{H}=-\frac{\kappa}{2}(\rho+P)
$$

and, therefore, $P<-\rho$ (a "phantom" equation of state) is equivalent to $\dot{H}>0$. A regime with $\dot{H}>0$, due to non-minimal coupling, called superinflation, was studied already in the 1980s [53,54]. Minimally coupled scalar fields have $\rho=\frac{\dot{\phi}^{2}}{2}+V(\phi)$ and $P=\frac{\dot{\phi}^{2}}{2}-V(\phi)$; hence, the derivative $\dot{H}$ in Equation (22) gives:

$$
\dot{H}=-\kappa \dot{\phi}^{2} / 2 \leq 0
$$

By contrast, for a non-minimally coupled scalar field, it is:

$$
\begin{aligned}
\rho & =\frac{\dot{\phi}^{2}}{2}+V(\phi)+3 \xi H \phi(H \phi+2 \dot{\phi}) \\
P & =\frac{\dot{\phi}^{2}}{2}-V(\phi)-\xi\left[4 H \phi \dot{\phi}+2 \dot{\phi}^{2}+2 \phi \ddot{\phi}+\left(2 \dot{H}+3 H^{2}\right) \phi^{2}\right]
\end{aligned}
$$

and $\dot{H}>0$ is a possibility. Indeed, exact solutions exhibiting explicitly this super-acceleration have been found in the context of early universe inflation [55,56] and of present-day quintessence [57].

Technically speaking, the non-minimally coupled scalar field action:

$$
S_{N M C}=\int d^{4} x \sqrt{-g}\left[\left(\frac{1}{2 \kappa}-\frac{\xi}{2} \phi^{2}\right) R-\frac{1}{2} \nabla^{\mu} \phi \nabla_{\mu} \phi-V(\phi)\right]
$$

is a scalar-tensor action, and gauge-independent formalisms have been developed to study cosmological perturbations in this class of theories. One can fix a gauge and proceed to study perturbations in that gauge, but there is the risk that the results are unphysical, an artifact of pure gauge modes. Indeed, the gauge-dependence problem plagued the early studies of cosmological perturbations produced during inflation. An alternative is to identify gauge-invariant variables and derive equations for these gauge-invariant quantities that assume the same form in all gauges. When this is done, a gauge-invariant formalism is obtained, which has the advantage of being completely gauge-independent and the disadvantage that the gauge-invariant variables are not physically transparent-they can receive a physical interpretation once a gauge is fixed. The original gauge-invariant formalism, due to Bardeen [58], has been refined over the years and was designed for cosmology in the context of general relativity. Here, we adopt the Bardeen-Ellis-Bruni-Hwang formalism [58-61], that is, a version of the 
Bardeen formalism [58], refined by Ellis, Bruni and Hwang and adapted by Hwang to a wide class of theories of gravity alternative to general relativity [62-68]. In fact, non-minimally coupled scalar field theory is a special case of scalar-tensor gravity, as can be seen by tracing in reverse the path outlined in Section 2, and it is straightforward to apply Hwang's formalism to this theory. The application of this formalism to non-minimally coupled inflation was reviewed in [69]. Slow-roll inflation with de Sitter universes as attractors in phase space is possible.

There are four slow-roll parameters, as opposed to the two of minimally coupled inflation (for comparison, $-\epsilon_{1}$ and $-\epsilon_{2}$ coincide with the usual parameters, $\epsilon$ and $\eta$, of minimally coupled inflation) [62-69]:

$$
\begin{aligned}
& \epsilon_{1}=\frac{\dot{H}}{H^{2}}, \quad \epsilon_{2}=\frac{\ddot{\phi}}{H \dot{\phi}} \\
& \epsilon_{3}=-\frac{\xi \kappa \phi \dot{\phi}}{H\left[1-\left(\frac{\phi}{\phi_{1}}\right)^{2}\right]}, \quad \epsilon_{4}=-\frac{\xi(1-6 \xi) \kappa \phi \dot{\phi}}{H\left[1-\left(\frac{\phi}{\phi_{2}}\right)^{2}\right]}
\end{aligned}
$$

(with $\phi_{1,2}$ constants), and $\epsilon_{4}$ vanishes for $\xi=1 / 6$. The spectral indices of scalar and tensor perturbations in the slow-roll approximation are then [62-68]:

$$
\begin{aligned}
& n_{S}=1+2\left(2 \epsilon_{1}-\epsilon_{2}+\epsilon_{3}\right) \\
& n_{T}=2\left(2 \epsilon_{1}-\epsilon_{3}\right)
\end{aligned}
$$

There is a possible signature of conformal coupling in the cosmic microwave background sky. In an inflationary super-acceleration regime, $\dot{H}>0$ (which is impossible with minimal coupling and realistic scalar field potentials), it is $\epsilon_{1}>0$, and one can obtain a blue spectrum of gravitational waves, $n_{T}>0$. Blue spectra of tensor perturbations are impossible in the standard scenarios of inflation (they are, however, possible in certain non-inflationary scenarios) with $\xi=0$ (for which $n_{T}=4 \dot{H} / H \leq 0$ ). More power is shifted to small wavelengths in comparison with minimally coupled inflation, which is interesting for the gravitational wave community, because it increases the chance of detecting cosmological gravitational waves with future space-based interferometers.

\section{Conclusions}

Supporting the idea that the inflaton field is conformally, rather than minimally, coupled is actually a pretty conservative view. Not doing so means allowing for a possible pathology in the local propagation of the inflaton, i.e., the possibility that this field propagates along the light cone when it is massive. This problem is even more serious during inflation because, in slow-roll, the cosmic dynamics are close to a de Sitter attractor for which $R$ is constant, and one could even have the causal pathology mentioned above (or be very close to it) at every spacetime point. This would indeed be a radical departure from known physics, which cannot be justified. The only way out of this conundrum is if $\xi=1 / 6$, and inflationary scenarios should be adapted to this constraint. 


\section{Acknowledgments}

It is a pleasure to thank Sebastiano Sonego for his leading role in the investigation of non-minimal coupling long ago, which led to the development of this project. We thank also three referees for useful remarks. This work is supported by the Natural Sciences and Engineering Research Council of Canada.

\section{Conflict of Interest}

The author declares no conflict of interest.

\section{References}

1. Brandenberger, R.H. Cosmology of the very early universe. AIP Conf. Proc. 2010, 1268, 3-70.

2. Cai, Y.-F.; Brandenberger, R.H.; Peter, P. Anisotropy in a non-singular bounce. Class. Quantum Grav. 2013, 30, 075019:1-075019:20.

3. Steinhardt, P.J.; Turok, N. Cosmic evolution in a cyclic universe. Phys. Rev. D 2002, 65, 126003:1-126003:20.

4. Khoury, J.; Ovrut, B.A.; Steinhardt, P.J.; Turok, N. Ekpyrotic universe: Colliding branes and the origin of the hot big bang. Phys. Rev. D 2001, 64, 123522:1-123522:23.

5. Khoury, J.; Steinhardt, P.J. Adiabatic ekpyrosis: Scale-invariant curvature perturbations from a single scalar field in a contracting universe. Phys. Rev. Lett. 2010, 104, 91301:1-91301:4.

6. Brandenberger, R.H. String Gas Cosmology. In String Cosmology; Erdmenger, J., Ed.; John Wiley and Sons: Hoboken, NJ, USA, 2009.

7. Battefeld, T.; Watson, S. String gas cosmology. Rev. Mod. Phys. 2006, 78, 435-454.

8. Brandenberger, R.H. String gas cosmology: Progress and problems. Class. Quantum Grav. 2011, 28, 204005:1-204005:14.

9. Chernikov, N.A.; Tagirov, E.A. Quantum theory of scalar field in de Sitter space. Ann. Inst. H. Poincaré A 1968, 9, 109-141.

10. Callan, C.G., Jr.; Coleman, S.; Jackiw, R. A new improved energy-momentum tensor. Ann. Phys. 1970, 59, 42-73.

11. Birrell, N.D.; Davies, P.C. Quantum Fields in Curved Space; Cambridge University Press: Cambridge, UK, 1980.

12. Birrell, N.D.; Davies, P.C. Conformal-symmetry breaking and cosmological particle creation in $\lambda \varphi^{4}$ theory. Phys. Rev. D 1980, 22, 322-329.

13. Nelson, B.; Panangaden, P. Scaling behavior of interacting quantum fields in curved spacetime. Phys. Rev. D 1982, 25, 1019-1027.

14. Ford, L.H.; Toms, D.J. Dynamical symmetry breaking due to radiative corrections in cosmology. Phys. Rev. D 1982, 25, 1510-1518.

15. Ishikawa, J. Gravitational effect on effective potential. Phys. Rev. D 1983, 28, 2445-2454.

16. Parker, L.; Toms, D.J. Renormalization-group analysis of grand unified theories in curved spacetime. Phys. Rev. D 1985, 29, 1584-1608. 
17. Sonego, S.; Faraoni, V. Coupling to the curvature for a scalar field from the equivalence principle. Class. Quantum Grav. 1993, 10, 1185-1187.

18. DeWitt, B.S.; Brehme, R.W. Radiation damping in a gravitational field. Ann. Phys. 1960, 9, 220-259.

19. Friedlander, F.G. The Wave Equation on a Curved Spacetime; Cambridge University Press: Cambridge, UK, 1975.

20. Hadamard, J. Lectures on Cauchy's Problem in Linear Partial Differential Equations; Dover: New York, NY, USA, 1952.

21. John, R.W. The Hadamard construction of Green's functions on a curved space-time with symmetries. Annalen der Physik 1987, 44, 531-544.

22. Will, C.M. Theory and Experiment in Gravitational Physics, 2nd ed.; Cambridge University Press: Cambridge, UK, 1993.

23. Gérard, J.-M. The strong equivalence principle from a gravitational gauge structure. Class. Quantum Grav. 2007, 24, 1867-1877.

24. Sotiriou, T.P.; Liberati, S.; Faraoni, V. Theory of gravitation theories: A no-progress report. Int. J. Mod. Phys. D 2008, 17, 399-423.

25. Faraoni, V. Cosmology in Scalar-Tensor Gravity; Kluwer Academic: Dordrecht, The Netherlands, 2004.

26. Buchbinder, I.L. Renormalization of quantum field theory in curved space-time and renormalization group equations. Fortschr. Phys. 1986 34, 605-628.

27. Odintsov, S.D. Renormalization group, effective action and grand unification theories in curved space-time. Fortschr. Phys. 1991, 39, 621-641.

28. Muta, T.S.; Odintsov, S.D. Model dependence of the nonminimal scalar graviton effective coupling constant in curved space-time. Mod. Phys. Lett. A 1991, 6, 3641-3646.

29. Elizalde, E.; Odintsov, S.D. Renormalization-group improved effective potential for finite grand unified theories in curved spacetime. Phys. Lett. B 1994, 333, 331-336.

30. Buchbinder, I.L.; Odintsov, S.D.; Shapiro, I.L. Effective Action in Quantum Gravity; IOP Publishing: Bristol, UK, 1992.

31. Buchbinder, I.L.; Odintsov, S.D.; Lichtzier, I. The behavior of effective coupling constants in "finite" grand unification theories in curved space-time. Class. Quantum Grav. 1989, 6, 605-610.

32. Bonanno, A. Coarse graining and renormalization group in the Einstein Universe. Phys. Rev. D 1995, 52, 969-980.

33. Bonanno, A.; Zappalá, D. Nonperturbative renormalization group approach for a scalar theory in higher-derivative gravity. Phys. Rev. D 1997, 55, 6135-6146.

34. Futamase, T.; Maeda, K. Chaotic inflationary scenario of the universe with a nonminimally coupled “inflaton” field. Phys. Rev. D 1989, 39, 399-404.

35. Futamase, T.; Rothman, T.; Matzner, R. Behavior of chaotic inflation in anisotropic cosmologies with nonminimal coupling. Phys. Rev. D 1989, 39, 405-411.

36. Kolb, E.W.; Salopek, D.; Turner, M.S. Origin of density fluctuations in extended inflation. Phys. Rev. D 1990, 42, 3925-3935. 
37. Fakir, R.; Unruh, W.G. Improvement on cosmological chaotic inflation through nonminimal coupling. Phys. Rev. D 1990, 41, 1783-1791.

38. Makino, N.; Sasaki, M. The density perturbation in the chaotic inflation with nonminimal coupling. Prog. Theor. Phys. 1991, 86, 103-118.

39. Barroso, A.; Casasayas, J.; Crawford, P.; Moniz, P.; Nunes, A. Inflation in the presence of a nonminimal coupling. Phys. Lett. B 1992, 275, 264-272.

40. Fakir, R.; Habib, S. Quantum fluctuations with strong curvature coupling. Mod. Phys. Lett. A 1993, 8, 2827-2842.

41. Laycock, A.M.; Liddle, A.R. Extended inflation with a curvature coupled inflaton. Phys. Rev. D 1994, 49, 1827-1839.

42. Garcia-Bellido, J.; Linde, A. Stationary solutions in Brans-Dicke stochastic inflationary cosmology. Phys. Rev. D 1995, 52, 6730-6738.

43. Komatsu, E.; Futamase, T. Constraints on the chaotic inflationary scenario with a nonminimally coupled "inflaton" field from the cosmic microwave background radiation anisotropy. Phys. Rev. D 1998, 58, 023004:1-023004:8.

44. Bassett, B.; Liberati, S. Geometric reheating after inflation. Phys. Rev. D 1998, 58, 021302(R):1-021302(R):5.

45. Futamase, T.; Tanaka, M. Chaotic inflation with running nonminimal coupling. Phys. Rev. D 1999, 60, 063511:1-063511:6.

46. Komatsu, E.; Futamase, T. Complete constraints on a nonminimally coupled chaotic inflationary scenario from the cosmic microwave background. Phys. Rev. D 1999, 59, 064029:1-064029:7.

47. Lee, J.; Koh, S.; Park, C.; Sin, S.J.; Lee, C.H. Oscillating inflation with a nonminimally coupled scalar field. Phys. Rev. D 1999, 61, 027301:1-027301:3.

48. Cooper, F.; Venturi, G. Cosmology and broken scale invariance. Phys. Rev. D 1981, 24, 3338-3340.

49. Kallosh, R.; Kofman, L.; Linde, A.D.; Van Proeyen, A. Superconformal symmetry, supergravity and cosmology. Class. Quantum Grav. 2004, 17, 4269-4338.

50. Basak, A.; Bhatt, J.R. A note on inflationary scenario with non-minimal coupling. ArXiv E-Prints, 2013, arXiv:1208.3298.

51. Gunzig, E.; Saa, A.; Brenig, L.; Faraoni, V.; Rocha-Filho, T.M.; Figueiredo, A. Superinflation, quintessence, and nonsingular cosmologies. Phys. Rev. D 2001, 63, 067301:1-067301:4.

52. Faraoni, V. Phase space geometry in scalar-tensor cosmology. Ann. Phys. 2005, 317, 366-382.

53. Lucchin, F.; Matarrese, S. Power-law inflation. Phys. Rev. D 1985, 32, 1316-1322.

54. Pollock, M.D. On the initial conditions for super-exponential inflation. Phys. Lett. B 1988, 215, 635-641.

55. Rocha Filho, T.M.; Figueiredo, A.; Brenig, L.; Gunzig, E.; Faraoni, V. Explicit analytic solutions of classical scalar field cosmology. Int. J. Theor. Phys. 2000, 39, 1933-1961.

56. Faraoni, V. Superquintessence. Int. J. Mod. Phys. D 2002, 11, 471-482.

57. Faraoni, V. Possible end of the universe in a finite future from dark energy with $w<-1$. Phys. Rev. D 2003, 68, 063508:1-063508:5.

58. Bardeen, J.M. Gauge-invariant cosmological perturbations. Phys. Rev. D 1980, 22, 1882-1905. 
59. Ellis, G.F.R.; Bruni, M. Covariant and gauge-invariant approach to cosmological density fluctuations. Phys. Rev. D 1989, 40, 1804-1818.

60. Ellis, G.F.R.; Hwang, J.-C.; Bruni, M. Covariant and gauge-independent perfect-fluid Robertson-Walker perturbations. Phys. Rev. D 1989, 40, 1819-1826.

61. Ellis, G.F.R.; Bruni, M.; Hwang, J.-C. Density-gradient-vorticity relation in perfect-fluid Robertson-Walker perturbations. Phys. Rev. D 1990, 42, 1035-1046.

62. Hwang, J.-C. Cosmological perturbations in generalized gravity theories: Solutions. Phys. Rev. D 1990, 42, 2601-2606.

63. Hwang, J.-C. Unified analysis of cosmological perturbations in generalized gravity. Phys. Rev. D 1996, 53, 762-765.

64. Hwang, J.-C. Cosmological perturbations in generalized gravity theories: Conformal transformation. Class. Quantum Grav. 1997, 14, 1981-1991.

65. Hwang, J.-C. Quantum generations of cosmological perturbations in generalized gravity. Class. Quantum Grav. 1998, 14, 3327-3336.

66. Hwang, J.-C. Gravitational wave spectra from pole-like inflations based on generalized gravity theories. Class. Quantum Grav. 1998, 15, 1401-1413.

67. Hwang, J.-C.; Noh, H. Density spectra from pole-like inflations based on generalized gravity theories. Class. Quantum Grav. 1998, 15, 1387-1400.

68. Hwang, J.-C.; Noh, H. Cosmological perturbations in generalized gravity theories. Phys. Rev. D 1996, 54, 1460-1473.

69. Faraoni, V. A crucial ingredient of inflation. Int. J. Theor. Phys. 2001, 40, 2259-2294.

(c) 2013 by the author; licensee MDPI, Basel, Switzerland. This article is an open access article distributed under the terms and conditions of the Creative Commons Attribution license (http://creativecommons.org/licenses/by/3.0/). 